\title{
Neuroprotective Potential of Sodium Orthovanadate in a Mouse Model of Chronic Unpredictable Mild Stress.
}

\author{
Angel Joshi \\ Panjab University Institute of Pharmaceutical Sciences \\ Ansab Akhtar \\ Panjab University Institute of Pharmaceutical Sciences \\ Anurag Kuhad \\ Panjab University Institute of Pharmaceutical Sciences \\ Sangeeta Pilkhwal Sah ( $\nabla$ spilkhwal@rediffmail.com ) \\ Panjab University Institute of Pharmaceutical Sciences
}

\section{Research Article}

Keywords: Depression, CUMS, sodium orthovanadate, BDNF, oxidative stress, corticosterone

Posted Date: May 19th, 2021

DOI: https://doi.org/10.21203/rs.3.rs-498351/v1

License: (c) (1) This work is licensed under a Creative Commons Attribution 4.0 International License. Read Full License 


\section{Abstract}

Depression is a psychiatric disorder characterized by low-esteem, anhedonia, social deficit, and lack of interest. Decreased BDNF and impaired TrKB signaling have been found to be associated in depression. Moreover, oxidative stress in the brain and subsequent reduction of BDNF have a prominent role in psychiatric disorders. In our study, depressive-like behavior was induced in mice by chronic unpredictable mild stress (CUMS) model. Further, sodium orthovanadate (SOV), a protein tyrosine phospahatase was used as a test drug as it is reported to stimulate BDNF levels. Sodium orthovanadate (SOV-5 mg/kg, $10 \mathrm{mg} / \mathrm{kg}$ ) and fluoxetine $(10 \mathrm{mg} / \mathrm{kg}$ ) were given to mice orally for 21 days prior 30 minutes of stress induction. Various behavioral studies like tail suspension test (TST), open field test (OFT), and sucrose preference test (SPT); biochemical analyses for corticosterone, reduced glutathione (GSH), lipid peroxidation (LPO), superoxide dismutase (SOD), nitric oxide (NO) and ELISA for BDNF were performed. Body weight was measured on a weekly basis. The behavioral tests reflected depressive-like behavior in CUMS, which was attenuated by SOV and fluoxetine. SOV $(10 \mathrm{mg} / \mathrm{kg})$ significantly decreased malondialdehyde levels, NO, whereas increased GSH and SOD in both the cortex and hippocampus. Besides, ELISA revealed the elevation of BDNF levels in the treatment groups compared with the disease group (CUMS). Therefore, the treatment with SOV appeared to reverse both oxidative and nitrosative stress. Elevated BDNF level was associated with attenuation of depressive-like behaviors and serum corticosterone levels. The findings of this preliminary study indicates that SOV has potential to restore mood and social interaction in depression and so further molecular mechanisms will be warranted for clinical translation.

\section{Highlights}

- CUMS produced depressive-like behaviors resembling depression in mice.

- Locomotor activity in OFT was impaired in mouse model CUMS.

- SOV attenuated depressive-like behaviors in mouse model of CUMS.

- SOV alleviated oxidative stress and decreased corticosterone levels.

- BDNF levels were elevated by SOV in mouse model of CUMS.

\section{Introduction}

Major Depressive Disorder (MDD) is one of the most prevalent, recurrent, and debilitating psychopathology forms. Epidemiological surveys indicate that the lifetime prevalence of MDD is $16.6 \%$, with estimates as high as $21.3 \%$ in women (LeMoult and Gotlib 2019).

The monoamine hypothesis formulated in the 1990s suggested deficiency or imbalances in the monoamine neurotransmitters, such as serotonin (5-HT), dopamine (DA), and norepinephrine (NE), as the cause of depression. The antidepressants are prescribed to treat mild to severe depression. However, despite the increased synaptic content of monoamine neurotransmitters, tricyclic antidepressants and selective serotonin reuptake inhibitors produce their effect after a lag period. Moreover, they are useful in only $50 \%$ of patients. Approximately $30-50 \%$ of patients don't even respond to their initial antidepressant trial, and the remission rates are as low as 37.5\% (Bousman, Arandjelovic et al. 2019). This phenomenon probably results from the complex and multifactorial MDD etiology, which comprises psychosocial, biological, environmental, and 
genetic factors, explaining why most patients fail to respond to the standard monoaminergic antidepressants (de Sousa, V Zanetti et al. 2015). This limitation led to a paradigm shift towards the neurotrophin hypothesis, as depression is associated with neuronal atrophy and neuronal loss in specific brain regions in several clinical and preclinical studies (Duman, Malberg et al. 2000, Mehrpouya, Nahavandi et al. 2015).

Considering neurotrophins, the brain-derived neurotrophic factor (BDNF) is the major neurotrophin present in the central nervous system, which regulates neurogenesis (Numakawa, Odaka et al. 2017, Bai, Zhu et al. 2012) as it has a prominent role in the growth, differentiation, maturation, and survival of neurons. It also promotes the formation of dendritic spines and thus improves transmision efficiency of synapses by increasing their number. Hence it is vital for synaptic plasticity and augmentation of neurotransmission (Bai, Zhu et al. 2012). BDNF triggers the intracellular downstream signaling via multiple pathways i.e., phosphatidylinositol 3-kinase

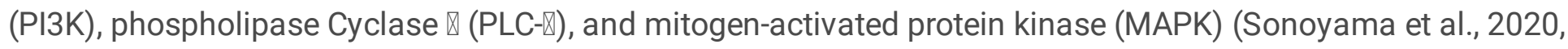
(Bai, Zhang et al. 2019), (Minichiello, Calella et al. 2002). This results in the activation of cAMP response element-binding protein (CREB), modulating the expression of BDNF levels (Feng, Wang et al. 2019), thus improving synaptic plasticity and cell survival.

A number of clinical and preclinical studies have implicated the close association between BDNF and the mood disorders. It has been implicated that in depression, the BDNF-TrkB pathway gets impaired, resulting in the reduced secretion of BDNF (Nagahara and Tuszynski 2011, Marshall, Zhou et al. 2018), and the studies reflect that the treatment with antidepressant consequently increases the BDNF levels (Wolkowitz, Wolf et al. 2011). Therefore, BDNF has a stimulatory action on neurons by its interaction with high-affinity TrkB receptors at tyrosine residues. Stressful life events trigger HPA axis hyperactivation in about $70 \%$ of depressive patients (Yang, Zhao et al. 2015). Increased corticosterone levels associated with chronic stress (McGill, Bundle et al. 2006) impairs hippocampal BDNF function, which supports the hippocampal atrophy reported in major depression (Jacobsen and Mørk 2006). Stress also promulgate the production of proinflammatory cytokines in the brain microglia, resulting in reduced hippocampal neurogenesis (Belleau, Treadway et al. 2019). The inflammatory cytokines affect the phosphorylation of BDNF receptor (TrkB), thereby interfering with BDNF signaling (Cortese, Barrientos et al. 2011, Frühauf-Perez, Temp et al. 2018).

Notably, sodium orthovanadate (SOV) is an inorganic compound belonging to the vanadium family, having a role as protein tyrosine phosphatase inhibitor (PTP) (Cherry, Calder et al. 2017). Protein tyrosine phosphatase inhibition by SOV results in the activation of the PI3K/AKT and MAPK pathway, which further stimulates BDNF levels (Wang, Lu et al. 2015), improves cell survival, synaptic plasticity, and delay neuronal damage (Kawano, Fukunaga et al. 2001). Additionally, a study done on subarachnoid hemorrhage in rats has revealed that the administration of SOV resulted in the elevation of the BDNF levels, and SOV protected cortical and hippocampal neurons after experimental subarachnoid hemorrhage by increasing BDNF (Hasegawa, Suzuki et al. 2011). Also, sodium orthovanadate elicits an antioxidant property by regulating levels of SOD, GPx, catalase, LPO, and glutathione in the diabetic rat (Sekar, Kanthasamy et al. 1990).

With the above background, depicting the role of BDNF-Trk signaling in major depressive disorder and the potential of SOV in improving BDNF signaling, we hypothesized that SOV can have a beneficial effect which was evaluated in CUMS induced rodent model of depression. 


\section{Material And Methods}

\subsection{Experimental animals}

Male Balb/c male mice with a weight ranging between 29-34 g were procured from Central Research Institute Kasauli and Central Animal House of Panjab University Chandigarh, India. All the mice were housed in a room at temperature $25 \pm 2^{\circ} \mathrm{C}$ with proper light-dark periods with water and food provided ad libitum. Whole interventional protocols were conducted between the period from 09:00 to 17:00 according to the guidelines provided by the Committee for the Purpose of Control and Supervision of Experiments on Animals (CPCSEA). The study protocol was approved by the Institutional Animal Ethical Committee (IAEC) of Panjab University, Chandigarh, with an approval number of PU/45/99/CPCSEA/IAEC/2019/303.

\subsection{Drugs and treatments}

Sodium orthovanadate (SOV) (catalog no.: S6508-10G) and fluoxetine (FLX) were procured from Sigma-Aldrich (St. Louis, MO, United States). Distilled water was used to dissolve SOV and administered orally with the help of an oral gavage. Normal saline, i.e., $0.9 \% \mathrm{NaCl}$, was used to dissolve the fluoxetine and then given orally in a dose of $10 \mathrm{mg} / \mathrm{kg}$ (Machado, Cunha et al. 2012). Distilled water was used as the solvent in the preparation of SOV and administered orally. Doses were decided in accordance with the previously reported studies (Hasegawa, Suzuki et al. 2011,Akhtar, Bishnoi et al. 2020) which have shown an attenuation of neuronal death and oxidative damage along with alleviation of mitochondrial dysfunction. Mouse ELISA kit for BDNF estimation was procured from Elabscience (catalog no.: E-EL-M0203), USA. The whole treatment was given daily starting from day 8 th to 28 th day.

\subsection{Experimental design}

Animals $(n=42)$ were randomly divided into seven groups, containing an equal number of animals $(n=6)$ as dipicted in Table 1.

Initially, for 1 st week, normal saline water $(0.9 \% \mathrm{NaCl})$ was given to the control group (that was not subjected to CUMS) and the CUMS group. From day 8th, treatment was given to all the groups prior 30 minutes of stress induction (CUMS group and per-se group) till 28th day. On 29th and 30th day sucrose preference test (SPT) was performed, whereas a tail suspension test (TST) was performed on the 31 st day. To check the effect on locomotor activity, open field test (OFT) was performed on 32nd day. The duration of the protocol lasted for 33 days from the induction of CUMS model until the sacrifice of animals and body weight was analyzed weekly. On the 33rd day, blood was collected from the animals under anesthesia, and then animals were euthanized for isolation of cortex and hippocampus to perform biochemical analysis and ELISA (Fig. 1). 
Table 1

Allocation of animals in the groups

\begin{tabular}{|llll|}
\hline Sr. No. & Groups & No. of Animals & Duration of Treatment \\
\hline 1. & Normal Control & 6 & 1 st -28 th \\
\hline 2. & Disease (CUMS) & 6 & 1 st -28 th \\
\hline 3. & CUMS + SOV $(5 \mathrm{mg} / \mathrm{kg} \mathrm{p.o.)}$ & 6 & 8th -28 th \\
\hline 4. & CUMS + SOV $(10 \mathrm{mg} / \mathrm{kg}$ p.o. $)$ & 6 & 8th -28 th \\
\hline 5. & CUMS + FLX $(10 \mathrm{mg} / \mathrm{kg}$ p.o. $)$ & 6 & 8th -28 th \\
\hline 6. & CUMS $+[S O V(5 \mathrm{mg} / \mathrm{kg})+\mathrm{FLX}(10 \mathrm{mg} / \mathrm{kg})]$ & 6 & 8th -28 th \\
\hline 7. & SOV $(10 \mathrm{mg} / \mathrm{kg} \mathrm{p.o.})$ & 6 & 8th -28 th \\
\hline
\end{tabular}

\subsection{Procedure for CUMS}

Animal stress models are commonly used in the preclinical antidepressant evaluation. Chronic unpredictable mild stress (CUMS) has been widely used in animals to mimic depressive-like behavior and is regarded as being close to the unexpected stressors of everyday life in humans. Animals were exposed to CUMS for 28 days except control and per-se group. The different types of stressors were given to the animal both on a regular and repetition basis till the sacrifice of animals as depicted in the Table 2 (Nollet, Guisquet et al. 2013).

Table 2

Schedule of CUMS

\begin{tabular}{|llll|}
\hline Sr. No. & Days & Stressors & Duration \\
\hline 1 & Day 1 & Food + Water Deprivation & $24 \mathrm{~h}$ \\
\hline 2 & Day 2 & Empty bottle + Foreign Object & $1 \mathrm{~h}+23 \mathrm{~h}$ \\
\hline 3 & Day 3 & Forced Swim* + Overnight illumination & $6 \mathrm{~min}+12 \mathrm{~h}$ \\
\hline 4 & Day 4 & Restraint stress + cage tilt** & $2 \mathrm{~h}+7 \mathrm{~h}$ \\
\hline 5 & Day 5 & Predator Sound + Food deprivation & $10 \mathrm{~min}+24 \mathrm{~h}$ \\
\hline 6 & Day 6 & Water deprivation + Overnight illumination & $24 \mathrm{~h}+12 \mathrm{~h}$ \\
\hline 7 & Day 7 & Cage tilt** + Empty bottle & $7 \mathrm{~h}+1 \mathrm{~h}$ \\
\hline
\end{tabular}

* Forced swim was done at water temperature of about $10-12^{\circ} \mathrm{C}$ for $8-10 \mathrm{~min}$.

** Cage was tilted or inclined at $45^{\circ}$.

\subsection{Behavioral studies}

\subsubsection{Open field test (OFT)}


To find out any effect of the drug on locomotor activity, OFT was conducted. The number of lines crossed was evaluated in the open field paradigm under normal daylight. The whole procedure was performed as per the previously described study (Idayu, Hidayat et al. 2011). Before the commencement of OFT, mice were acclimatized to environment for 2-3 min. The test apparatus consisted of an arena with a measurement of 50 $\mathrm{cm} \times 30 \mathrm{~cm}$ and painted with black colour. The floor of the test apparatus consisted of 25 virtually produced grids made with the help of Ethovision. Each mouse was placed in the middle of the arena and then allowed to explore freely. The number of lines crossed by the mice with all their paws within each grid in 6 min was evaluated. After each testing, the apparatus was cleaned with $70 \%$ ethanol to remove any odor and clues of previous mouse made by its urine and fecal content. Results were expressed as number of lines crossed.

\subsubsection{Sucrose preference test (SPT)}

A state of anhedonia characterizes depression, and lowered sucrose consumption in rodents is a clear-cut indication of this state. The procedure was performed as described previously (Wang, Cui et al. 2011). Briefly, $30 \mathrm{~h}$ before the test, mice were deprived of water and food, then 2 bottles were placed in the cage containing $1 \%$ sucrose solution (w/v) and regular tap water, respectively. Animals were freely allowed to access both the bottles for $24 \mathrm{~h}$. At the end of $24 \mathrm{~h}$, the sucrose preference (\%) was calculated as below. Results were expressed as the percentage of sucrose consumed.

\section{Sucrose consumption \\ Sucrose consumption + Water consumption \\ $\mathrm{X} 100$ \\ 2.5.3 Tail suspension test (TST)}

This test was performed to assess learned helplessness in mice as per the previously described procedure (Kulkarni and Dhir 2007). Prior to conduction of TST, all mice were acclimatized to surroundings for 2-3 min. Mice were isolated from any external sound and visuals and placed $50-55 \mathrm{~cm}$ above the ground by fixing the tail on the frontal lever (approx. $1 \mathrm{~cm}$ from tip) with adhesive tape. During the total $6 \mathrm{~min}$ of test, acclimatization was done for initial 2 min, and the remaining time was utilized for recording the immobility of mice on kymograph. Results were expressed as immobility time (sec).

\subsubsection{Body weight measurement}

Animal body weight was measured every week of the experimental protocol. Fluctuation in body weight was calculated based on data acquired weekly. Results were expressed as grams.

\subsection{Biochemical estimations}

\subsubsection{Tissue homogenate preparation}

After completing all the behavioral evaluations, the animals were sacrificed under anesthesia with ketamine (70 $\mathrm{mg} / \mathrm{kg}$ ) and xylazine $(10 \mathrm{mg} / \mathrm{kg})$ combination. During anesthesia, the blood was collected through the retroorbital plexus and stored in the eppendorf containing EDTA. Afterward, the animals were sacrificed; brains were isolated and perfused with PBS. Cortex and hippocampus were isolated later from the whole brain. The isolated tissues were stored and homogenized in 10\% (w/v) homogenization buffer (comprising of $10 \mathrm{mM} \mathrm{Tris-}-\mathrm{HCl}, 150$ $\mathrm{mM} \mathrm{MgCl}_{2}, 1 \mathrm{mM}$ EDTA, $1 \%$ Triton X 100, pH equivalent to 7.4) and centrifuged at 10,000 rpm and $4^{\circ} \mathrm{C}$ for 20 
min. After the centrifugation, the supernatant was isolated by pipette and stored at $-80^{\circ} \mathrm{C}$ for various antioxidant assays and ELISA. Further, the plasma was separated by the process of centrifugation at 10,000 rpm for $10 \mathrm{~min}$ and stored at $-80^{\circ} \mathrm{C}$ for further estimations.

\subsubsection{Estimation of Protein}

The biuret method was used for the quantification of protein (Gornall, Bardawill et al. 1949). The standard curve of bovine serum albumin was used to determine protein concentration expressed in $\mathrm{mg} / \mathrm{ml}$. Thus, the values obtained were used in the calculations of other biochemical results.

\subsubsection{Reduced glutathione (GSH) assay}

Reduced glutathione was estimated based on a previous study (Jollow, Mitchell et al. 1974). In the method, 100 $\mu \mathrm{l}$ of the supernatant of tissue homogenate was added to $1 \mathrm{ml}$ of $4 \% \mathrm{w} / \mathrm{v}$ sulfosalicylic acid. The precipitate was formed, and the reaction mixture was kept at temperature $2-8^{\circ} \mathrm{C}$ in the refrigerator. After one hour, samples were centrifuged in a cold centrifuge, i.e., $4^{\circ} \mathrm{C}$, at a rotation of $1200 \mathrm{~g}$ for $15 \mathrm{~min}$. Pellet was discarded to obtain the supernatant. Furthermore, $100 \mu \mathrm{l}$ of this supernatant, $2.7 \mathrm{ml}$ of $0.1 \mathrm{M}$ phosphate buffer (pH 8), and $200 \mu \mathrm{l}$ of 0.1 M 5,5-dithiobis-2-nitrobenzoic acid (DTNB-Ellman's reagent) were mixed to produce a pale-yellow color. The color produced was read at $412 \mathrm{~nm}$ with a UV-visible spectrophotometer (Perkin Elmer, USA). The calculation was done by applying the molar extinction coefficient of $1.36 \times 10^{4} \mathrm{M}^{-1} \mathrm{~cm}^{-1}$, and results were expressed as $\mu \mathrm{M}$ GSH per mg protein.

\subsubsection{Estimation of lipid peroxidation (LPO)}

LPO was conducted by evaluating malondialdehyde levels (Wills 1965). Concisely, $0.5 \mathrm{ml}$ of tissue homogenate was added to $0.5 \mathrm{ml}$ of Tris- $\mathrm{HCl}$ followed by $2 \mathrm{~h}$ of incubation at $37^{\circ} \mathrm{C}$. To the above mixture, $1.0 \mathrm{ml}$ of $10 \%$ trichloroacetic acid (TCA), was added and then centrifuged at $1000 \mathrm{~g}$ for $10 \mathrm{~min}$. Then, $1.0 \mathrm{ml}$ of supernatant from the above solution was pipetted out and mixed with $1.0 \mathrm{ml}$ of thiobarbituric acid $(0.67 \% \mathrm{w} / \mathrm{v})$. The tubes containing the mixture were put in boiling water, for $10 \mathrm{~min}$, followed by cooling and addition of $1.0 \mathrm{ml}$ of double-distilled water. Reading for absorbance was noted at $532 \mathrm{~nm}$ (UV-VIS Spectrophotometer, Perkin Elmer, Lambda 20). Levels of MDA was measured and expressed as the amount of MDA (nmoles/mg protein).

\subsubsection{Estimation of superoxide dismutase (SOD)}

SOD was estimated as per described study (Kono 1978). $0.1 \mathrm{mM}$ EDTA at $10.8 \mathrm{pH}, 96 \mathrm{mM}$ nitro blue tetrazolium (NBT) and $50 \mathrm{mM}$ sodium carbonate mixture were prepared. Supernatant of tissue homogenate (50 $\mu \mathrm{l})$ was added to above mixture followed by hydroxylamine hydrochloride $(0.5 \mathrm{ml})$ results in the oxidation of hydroxylamine hydrochloride. Finally, absorbance was measured at $560 \mathrm{~nm}$ wavelength for $2 \mathrm{~min}$ and SOD values were calculated as SOD units/mg protein.

\subsubsection{Estimation of plasma corticosterone}

The plasma corticosterone estimation was done as previously described (Bartos and Pesez 1979). For the assessment of corticosterone levels in the blood (plasma), the reagents respectively reagent $A(0.10 \%$ p-nitroso$\mathrm{N}, \mathrm{N}$-dimethylaniline in ethanol), reagent $\mathrm{B}(0.10 \%$ phenol in ethanol), and reagent $\mathrm{C}(1 \%$ aqueous solution of potassium ferricyanide) were prepared freshly. An equal volume of ethanol and sample $(1 \mathrm{ml})$ were added to reagent $A$, and then the final solution was stored in ice water for 5 min only. Further, $0.5 \mathrm{ml}$ of $0.10 \mathrm{M} \mathrm{NaOH}$ was 
added and incubated for the duration of $5 \mathrm{~h}$ at $0^{\circ} \mathrm{C}$. Upon completing the above procedure, $2 \mathrm{ml}$ of buffer $(0.20$

$\mathrm{M}$ boric acid, $0.20 \mathrm{M} \mathrm{KCl}$, and $0.20 \mathrm{M} \mathrm{KOH}$ ) was added along with the addition of reagent $\mathrm{B}$ and reagent $\mathrm{C}$ in the resulted solution. Then, the final solution prepared was kept for $10 \mathrm{~min}$ at $20 \pm 2 \varangle \mathrm{C}$. Lastly, the reading was noted down at $650 \mathrm{~nm}$ (UV-VIS Spectrophotometer, Perkin Elmer, Lambda 20).

\subsubsection{Estimation of nitrite (NO)}

For nitrite assay, equal volumes, i.e., $100 \mu$ of tissue homogenate sample, was mixed with $100 \mu$ l of Griess reagent $(0.1 \%$ naphthyl ethylenediamine dihydrochloride $+1 \%$ sulphanilamide in $5 \%$ phosphoric acid). This mixture was incubated at $25-30^{\circ} \mathrm{C}$ for $10 \mathrm{~min}$ in a dark place. Absorbance readings were taken in a UV-visible spectrophotometer at $540 \mathrm{~nm}$. Nitrite concentrations were interpreted from a standard curve of sodium nitrite solution, and results were expressed as $\mu \mathrm{g}$ of nitrite per mg protein (Hasegawa, Suzuki et al. 2011).

\subsection{Molecular estimations}

\subsubsection{Enzyme-linked immunosorbent assay (ELISA)}

ELISA was performed in the hippocampal and cortical tissue of the brain as they are the major brain regions associated in mood disorders.

\subsubsection{BDNF protein}

BDNF has a significant role in the neurogenesis and synaptic plasticity. Levels of BDNF were analyzed using a mouse BDNF ELISA kit (Elabscience). $100 \mu \mathrm{l}$ of standard/tissue homogenate was added to each well of the precoated plates and incubated for $90 \mathrm{~min}$ at $37^{\circ} \mathrm{C}$. The liquid was removed, and $100 \mu \mathrm{l}$ of biotinylated detection antibody was added to each of the wells and incubated for $60 \mathrm{~min}$ at $37^{\circ} \mathrm{C}$. The plate was washed 3 times using wash buffer. Then $100 \mu$ of HRP conjugate was added to each of the wells and kept at $37^{\circ} \mathrm{C}$ for 30 min. The plate was then again washed 5 times, and $90 \mu$ of substrate reagent was added to it and incubated for $15-30 \mathrm{~min}$ at $37^{\circ} \mathrm{C}$ for blue color development. Finally, $50 \mu \mathrm{l}$ of stop solution was added to each well, and a color change to yellow was observed. The optical density of the reaction was measured at $450 \mathrm{~nm}$. Results were expressed as $\mathrm{pg} / \mathrm{ml}$ of BDNF.

\subsection{Statistical Analysis}

Analysis of data was done using a one-way ANOVA or two-way ANOVA followed by a Tukey's post hoc test or Bonferroni's post hoc test respectively for multiple comparisons. Statistically significant effects were defined as those with levels of P-values $<0.05$. The standard error of the mean was represented by error bars. Prism Graphpad 5.0 (GraphPad Software Inc., Ca, USA) was used to analyze the data.

\section{Results}

\subsection{Effect of SOV, fluoxetine and their combination on locomotor activity in open field test}


CUMS for 28 consecutive days significantly $(P<0.001)$ decreased the ambulatory score as compared to the control group. Treatment with SOV (10 mg/kg) and fluoxetine significantly increased the ambulatory score when compared to CUMS group $(P<0.05)$. Treatment with a combination group showed no significant difference in the ambulatory score than the CUMS and control group. The per-se group did not show any difference compared with the control group (Fig. 2).

\subsection{Effect of SOV, fluoxetine and their combination on the consumption of sucrose in Sucrose preference test}

The sucrose preference test was measured by percentage sucrose preference. Sucrose preference was significantly reduced in CUMS group compared to the control group $(P<0.001)$. Treatment with SOV $(5 \mathrm{mg} / \mathrm{kg}$ and $10 \mathrm{mg} / \mathrm{kg}$ ), fluoxetine, and combination groups significantly increased percentage sucrose preference as compared to CUMS group $(P<0.001)$. Fluoxetine was not showing any significant difference among treatment groups. Per-se group demonstrated a significant decrease in the sucrose consumption as compared to the control group $(P<0.001)$ (Fig. 3)

\subsection{Effect of SOV, fluoxetine and their combination on an immobility duration in tail suspension test}

Induction of depressive-like behavior by CUMS was evident by significant increase in the immobility time $(\mathrm{P}<$ $0.05)$ as compared to the control group. Treatment with sodium orthovanadate $(5 \mathrm{mg} / \mathrm{kg}$ and $10 \mathrm{mg} / \mathrm{kg})$ and fluoxetine significantly decreased the immobility time when compared to CUMS group $(P<0.01$ and $P<0.05)$ and effects were comparable. Treatment with the combination drug also significantly reduced the immobility time when compared with CUMS group $(P<0.01)$. Per-se group did not show any significant effect compared to the control group (Fig. 4).

\subsection{Effect of SOV, fluoxetine and their combination on body weight}

Body weight was observed to be significantly low in CUMS (diseased) group from day 8 to day 33 as compared to the control group $(P<0.001)$. Treatment with SOV $(5 \mathrm{mg} / \mathrm{kg}$ and $10 \mathrm{mg} / \mathrm{kg})$ produced no significant body weight change when compared with the CUMS group. However, fluoxetine treatment significantly increased body weight compared to CUMS group on day $15(P<0.05)$, on day 28 , and day $33(P<0.01)$. Similarly, combination treatment also significantly increased the body weight of CUMS rats as compared to CUMS group from day 22 to $33(P<0.001)$. The per-se group showed a significant decrease in body weight as compared to the control group from day 15 to $33(P<0.001)$ (Table 3$)$.

Table 3: Effect of sodium orthovanadate, fluoxetine and their combination on the body weight. 


\begin{tabular}{|c|c|c|c|c|c|c|c|}
\hline \multirow[t]{4}{*}{ DAYS } & \multicolumn{7}{|l|}{ GROUPS } \\
\hline & \multirow[t]{3}{*}{ Control } & \multirow{3}{*}{$\begin{array}{l}\text { Disease } \\
\text { (CUMS + } \\
\text { Vehicle) }\end{array}$} & \multirow{3}{*}{$\begin{array}{l}\text { CUMS } \\
+ \text { SOV } \\
(5 \mathrm{mg} / \mathrm{kg})\end{array}$} & \multirow{3}{*}{$\begin{array}{l}\text { CUMS + } \\
\text { SOV } \\
(10 \mathrm{mg} / \mathrm{kg})\end{array}$} & \multirow{3}{*}{$\begin{array}{l}\text { CUMS + } \\
\text { [(SOV } \\
(5 \mathrm{mg} / \mathrm{kg}) \\
+\mathrm{FLX} \\
(10 \mathrm{mg} / \mathrm{kg})]\end{array}$} & \multirow{3}{*}{$\begin{array}{l}\text { CUMS + } \\
\text { FLX } \\
(10 \mathrm{mg} / \mathrm{kg})\end{array}$} & \multirow[t]{3}{*}{$\begin{array}{l}\text { SoV } \\
(10 \mathrm{mg} / \mathrm{kg})\end{array}$} \\
\hline & & & & & & & \\
\hline & & & & & & & \\
\hline 1 & $33.50 \pm 0.8$ & $30.86 \pm 0.7$ & $29.94 \pm 1.5$ & $29.43 \pm 0.4$ & $31.02 \pm 0.9$ & $29.28 \pm 0.7$ & $29.14 \pm 0.8$ \\
\hline 8 & $34.50 \pm 0.6$ & $29.5 \pm 0.3^{*}$ & $30.23 \pm 1.6$ & $29.43 \pm 0.3$ & $33.12 \pm .17$ & $32.71 \pm 1.5$ & $31.42 \pm 0.4$ \\
\hline 15 & $37.50 \pm 1.2$ & $27.5 \pm 0.4^{*}$ & 27.08ะ1.5@ & $26.51 \pm 0.8 @$ & $31.76 \pm 2.3$ & $32.85 \pm 1.6^{\#}$ & $29.85 \pm 1.2^{*}$ \\
\hline 22 & $37.34 \pm 1.2$ & $28.5 \pm 1.3^{*}$ & $28.08 \pm 1.4$ & 26.57士1.6@ & $36.87 \pm 1.6^{\#}$ & $32.85 \pm 1.6$ & $27.71 \pm 1.3^{*}$ \\
\hline 28 & $35.34 \pm 0.8$ & $25.8 \pm 1.7^{*}$ & 26.75ะ1.1@ & 26.85×1.5@ & $41.56 \pm 1.7^{\#}$ & $37.14 \pm 1.5^{\#}$ & $26.57 \pm 1.7^{*}$ \\
\hline 33 & $39.35 \pm 1$ & $24.5 \pm 1.6^{*}$ & $30.75 \pm 1.6 \#$ \#@ & 29.14士1.3@ & $34.68 \pm 2.0^{\#}$ & $38.66 \pm 1.8^{\#}$ & $29.34 \pm 1.1^{*}$ \\
\hline
\end{tabular}

Values are expressed as mean \pm SEM. For statistical significance, ${ }^{*} \mathrm{p}<0.05$ as compared to the control group;

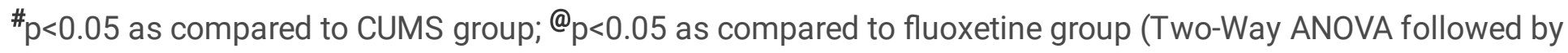
Bonferroni's post hoc test). CUMS: Chronic Unpredictable Mild Stress, SOV: Sodium Orthovanadate.

\subsection{Effect of SOV, fluoxetine and their combination on the level of GSH, LPO, and activity of SOD}

In both the hippocampus and cortex areas in CUMS group, the levels of GSH were decreased $(P<0.001)$ significantly compared to the control group. Treatment with sodium orthovanadate $(5 \mathrm{mg} / \mathrm{kg}$ and $10 \mathrm{mg} / \mathrm{kg}$ ) significantly increased the levels of GSH in both cortex $(P<0.05$ and $P<0.01)$ and hippocampus $(P<0.05)$. Fluoxetine showed a significant increase in the levels of GSH in both cortex and hippocampus compared to the CUMS group $(\mathrm{P}<0.05)$. Per-se group did not show any significant result in the GSH levels compared to the control group, both in the hippocampus and cortex (Fig. 5).

The level of LPO in CUMS group was found to be significantly increased in both cortex $(P<0.001)$ and hippocampus $(P<0.05)$ as compared to the control group. Treatment with sodium orthovanadate $(10 \mathrm{mg} / \mathrm{kg})$, fluoxetine, and combination group significantly decreased the levels of LPO as compared to CUMS group in both cortex $(P<0.01)$ and hippocampus $(P<0.05)$. In addition to the above, sodium orthovanadate $(5 \mathrm{mg} / \mathrm{kg})$ significantly decreased the levels of LPO in the cortex region $(P<0.001)$. In both cortex and hippocampus, the lipid peroxidation did not increase in the per-se group compared to the control group (Fig. 6).

The antioxidant enzyme SOD was significantly decreased in both the hippocampal and cortical regions of the brain in CUMS group compared to control mice $(P<0.001)$. Treatment with sodium orthovanadate $(10 \mathrm{mg} / \mathrm{kg})$ showed a significant increase in the level of SOD in both hippocampus $(P<0.01)$ and cortex $(P<0.05)$ as compared with CUMS group. Fluoxetine showed a significant increase in the SOD levels in the hippocampus as compared to CUMS group $(P<0.001)$, and also SOD levels in the combination group were found to be increased 


\subsection{Effect of SOV, fluoxetine and their combination on the levels of plasma corticosterone and NO}

The plasma corticosterone levels were significantly increased in CUMS group compared to the control group ( $P$ $<0.001)$. Treatment with sodium orthovanadate $(5 \mathrm{mg} / \mathrm{kg}$ and $10 \mathrm{mg} / \mathrm{kg})$, fluoxetine, and combination group significantly decreased the levels of plasma corticosterone as compared to CUMS group $(\mathrm{P}<0.001)$. The per-se group produced a significant decrease in plasma corticosterone levels as compared to the control group ( $\mathrm{P}<$ 0.001) (Fig. 8).

CUMS significantly increased nitrite levels in both cortex and hippocampus $(\mathrm{P}<0.001)$ compared to the control group. Treatment with SOV $(10 \mathrm{mg} / \mathrm{kg})$ significantly decreased the nitrite levels in both hippocampus $(\mathrm{P}<$ $0.001)$ and cortex $(P<0.05)$ as compared to CUMS group. Fluoxetine also showed a significant decrease in nitrite levels when compared to CUMS group in both cortex $(P<0.05)$ and hippocampus $(P<0.001)$ regions. However, SOV $(10 \mathrm{mg} / \mathrm{kg})$ in both cortex $(P<0.05)$ and hippocampus $(P<0.001)$ area significantly decreased NO levels as compared to CUMS group. Per-se group significantly increased the levels of nitrite as compared to the control group $(\mathrm{P}<0.001)$ (Fig. 9).

\subsection{Effect of SOV and fluoxetine on the levels of brain-derived neurotrophic factor}

Levels of BDNF were decreased significantly in both cortex and hippocampus compared to the control group $(P<0.01)$. Treatment with SOV $(5 \mathrm{mg} / \mathrm{kg}$ and $10 \mathrm{mg} / \mathrm{kg})$ and fluoxetine significantly increased the BDNF levels in both hippocampus and cortex as compared to CUMS group $(P<0.05)$ (Fig. 10).

\section{Discussion}

In our present study, we used sodium orthovanadate (SOV) to establish the role of BDNF and oxidative stress in depression. Moreover there is an inverse relationship between them, reflecting a possible mechanism of interaction between oxidative stress and neurotrophin dysfunction (Zhang, Chen et al. 2015). Corticosterone, a stress hormone, is an indicator of the anxiety and depressive- like behavior in an individual. Stress elevates corticosterone levels by activating the HPA axis, resulting in neuronal atrophy arising due to decreased brain levels of neurotrophins like BDNF. Corticosterone decreases BDNF mRNA expression gradually, resulting in diminished levels of BDNF protein translation (Liu, Walther et al. 2005). BDNF is one of the growth factors that trigger neuronal survival after BDNF-TrkB signaling. Impairment in the neurotrophins, mainly BDNF, leads to depressive-like behavior, increased hippocampal dendritic atrophy, cell death, and reduced LTP. Impaired BDNF expression has also been reported in MDD. A study has revealed that cortisol levels get elevated, and serotonin levels get declined on the induction of chronic stress via serotonin reuptake, further resulting in the depressivelike behavior (Sheikh, Hayden et al. 2010). Chronic stress causes HPA axis dysregulation, and many studies have reported a decrease in the proliferation and survival of hippocampal neurons when the HPA axis is 
dysregulated (Gomez, Calderón-Rivera et al. 2020). Morover chronic stress induced HPA axis dyfunctioning results in the production of proinflammatory cytokines (Belleau, Treadway et al. 2019). Neuroinflammation leads to oxidative stress and both of them together generate a vicious cycle resulting in reduced hippocampal neurogenesis. Therefore, considering this CUMS paradigm, which is a well-validated model of depression produced by the set of stressors in rodents (Schildkraut 1965), the present study was used to elucidate the impaired role of BDNF levels in CUMS induced depressive like behavior and its amelioration with SOV.

CUMS significantly downregulates the levels of BDNF and CREB, resulting in a depressive-like behavior (LeviMontalcini 1950). Increased corticosterone levels and decreased levels of BDNF after CUMS in our results are in line with the above findings. However, our study also revealed that the treatment with SOV significantly decreased the plasma corticosterone levels. The effect produced by SOV per-se on corticosterone levels is consistent with previous findings where vanadium compound attenuated corticosterone levels in rats (Katayama, Yamada et al. 2010). According to a prior study, the chronic FLX treatment in CUMS exposed rats also normalized the corticosterone levels (Karege, Vaudan et al. 2005) as reported in our study. ELISA was performed to predict the effect of FLX treatment on BDNF. It was observed that fluoxetine significantly increased the levels of BDNF in both hippocampus and cortex may be via triggering transcription regulator, i.e., CREB (Chen, Dowlatshahi et al. 2001).

Body weight indicates the pathogenesis of the disease, and it was found that CUMS rats demonstrated a significant decrease in weight compared to the control group, thus depicting one of the core signs of a depressive-like behavior (Babiec, Guglietta et al. 2014). In our study, treatment with SOV produced no significant gain in body weight compared to CUMS group. However, SOV per-se has made a significant decrease in weight gain as compared to the control group. The results support the previous finding in which vanadium-fed dams had lower food intakes and weight gains than controls during pregnancy (Ferré, Baler et al. 2009). Vanadium compound-induced weight loss could also be attributed to the reduction in neuropeptide $Y$ synthesis (NPY), which is responsible for the stimulation of appetite (Ayoub and Pfleger 2010). At the same time, significant weight gain was observed in the fluoxetine and control groups. In the former case, the antidepressant tends to increase body weight (Trifilieff, Rives et al. 2011), appetite, and a study conducted on humans also support our results regarding the elevation of body weight by fluoxetine as serotonin is responsible for appetite (Hern, Baig et al. 2010).

Increased immobility in tail suspension test (TST), regarded as a condition of 'failure to adapt to stress' (Hasbi, Fan et al. 2009) was produced after CUMS induction. Significant association exist between decreased immobility and the potency of antidepressants in tail suspension tests (Gomes, Ferreira et al. 2013).

CUMS model shows a declined sensitivity to reward, termed as the anhedonic state (Hutchinson, Chou et al. 2009), one of the core symptoms of depression. A study confirmed that mice exposed to CUMS consume less sucrose fluid (Fukuchi 2020), supporting our findings. An open field test was conducted to analyze the locomotor activity and explore the novel environment (Wills 1965). Many studies depict the decline in the number of line crossing activity (OFT) in CUMS group, reflecting a depressive-like behaviors (Fukuchi 2020). Our study also showed a similar finding reflecting the effect of CUMS in the induction of a depressive-like behaviors. However, both the SOV doses in the present study improved the core features of depression in rodents like anhedonia, despair behavior, and hypo-locomotion. SOV per-se resulted in decreased sucrose

Page 12/26 
consumption that could result from its roles in improving leptin and insulin signaling, which play an essential role in regulating energy balance through food-associated reward control (Zhang, Chen et al. 2015).

Hippocampal oxidative stress is induced due to low BDNF after chronic unpredictable mild stress. Numerous studies have highlighted that stress significantly decreases BDNF mRNA expression in the frontal cortex and hippocampus (Kim, Lee et al. 2019), thus indicating a link between low BDNF and oxidative stress. A study found that BDNF downregulates ethanol-induced cellular oxidative stress and apoptosis in developing hypothalamic neuronal cells (Frühauf-Perez, Temp et al. 2018) thus affirming antioxidant-like activities of BDNF (Mehrpouya, Nahavandi et al. 2015). In the present study, we analyzed MDA levels (an indicator of lipid peroxidation), NO, GSH, and SOD in the hippocampal and cortical tissues of mice exposed to CUMS. CUMS exposure resulted in the generation of oxidative stress and nitrosative stress in both the cortex and hippocampus. However, chronic SOV treatment ameliorated oxidative and nitrosative stress in the brain, emphasizing the role of BDNF in mediating antioxidant effects (Bibring 1953). SOV significantly increased SOD levels after CUMS exposure and the findings are consistent with previous study indicating neuroprotective effect of SOV. MDD is associated with lipid peroxidation and decreased antioxidative enzyme activities resulting in reactive oxygen species (ROS) generation (Schiller, Minkel et al. 2013; Kim, Lee et al. 2019). Neuronal degeneration is a significant consequence of ROS generation.

In vitro studies suggested that ROS act in a neurotoxic as well as in a neuroprotective manner, which is enhanced by TrkB (Harlow, Newcomb et al. 1986). However, ROS has a significant role in psychiatric disease due to the vulnerability of the central nervous system to oxidative stress and CUMS results in induction of ROS expression by Akt pathway modulation (Mulinari 2012). In addition to the above finding, a study conducted on cisplatin-induced ROS suggested that BDNF attenuates ROS generation, resulting in the decline in ROS levels (Carroll 1971). Indeed, a specific pathway has not been evolved fully to support the link between ROS and TrkB.

Meanwhile, a study conducted on cell lines revealed that vanadate compound per-se could generate ROS resulting in the decreased levels of SOD via MAPK pathway activation (Fawcett and Barkin 1997). And our study also implies that SOV per-se decreased SOD and increased nitrite levels per-se while it does not affect MDA levels. Whereas it produced a significant antioxidant effect in CUMS exposed rats supported by previously conducted study (Kim, Lee et al. 2019).

Our study revealed that CUMS exposure increased nitrite levels, whereas SOV (per-se) also increased nitrite levels via the Akt pathway's activation (Aid, Kazantseva et al. 2007) while SOV at a dose of $10 \mathrm{mg} / \mathrm{kg}$ significantly decreased its level. CUMS activates microglia, which gets activated to produce inflammatory cytokines. These inflammatory markers are the leading cause for the production of nitrites in the brain whereas protein tyrosine phosphatase 1B (PTP1B), a member of the protein tyrosine phosphatases (PTPs) family, positively regulates neuroinflammation by causing dephosphorylation of proteins at tyrosine residues. SOV, a PTP inhibitor reduces this feature induced by CUMS, resulting in a decline in nitrite levels than the CUMS group (Seifer, Feng et al. 2006).

A report suggests that PTP1B down-regulates the neuronal BDNF-TrkB pathway through the dephosphorylation of TrkB, whereas PTP1B inhibition boosts BDNF signaling (Zhang, Chen et al. 2015). It is well postulated that SOV, directly acting at tyrosine residues of TrkB, preserves its signaling and also recovers tyrosine kinase activity of TrkB by upregulating m-BDNF (Akhtar, Bishnoi et al. 2020). 


\section{Conclusion}

BDNF has many divergent roles in the neuroscience area, and its regulatory effect on the TrkB receptor opens avenues for further research on psychiatric and neurological disorders. PTP inhibitors positively modulate BDNF-TrKB signaling. In the present study study, SOV triggered BDNF levels at a dose of $10 \mathrm{mg} / \mathrm{kg}$ and prominently reversed the oxidative stress markers, reduced corticosterone levels and ameliorated depressivelike behaviors in the animals. In future studies, SOV could be taken as a potential candidate for neuroscience research due to its PTP-B1 inhibiting property.

\section{Declarations}

\section{Declaration of interest}

None.

\section{Funding}

This work is supported by All India Council for Technical Education (AICTE), New Delhi, Govt. of India.

\section{Conflict of interest}

The authors declare no conflict of interest.

\section{Availability of data and material}

Not Applicable

\section{Code availability}

Not Applicable

\section{Authors' contributions}

Angel Joshi has conducted the entire study and also written a whole manuscript. Ansab Akhtar has performed the editing of manuscript and support in the conduction of study. Anurag Kuhad and Sangeeta Pilkhwal Sah had reviewed, edited and finalized the manuscript. In addition to above, both Anurag Kuhad and Sangeeta Pilkhwal Sah had provide a technical support in my mentioned study. The authors declare that all data were generated in-house and that no paper mill was used.

\section{Ethics approval}

The study protocol was approved by the Institutional Animal Ethical Committee (IAEC) of Panjab University, Chandigarh, with an approval number of PU/45/99/CPCSEA/IAEC/2019/303.

\section{Consent to participate}

Not Applicable 


\section{Consent for publication}

Not Applicable

\section{Acknowledgment}

All India Council for Technical Education (AICTE), New Delhi, was thanked and acknowledged for providing a scholarship for the M. Pharmacy course.

\section{References}

1. Aid, T., A. Kazantseva, et al. (2007). "Mouse and rat BDNF gene structure and expression revisited." Journal of neuroscience research 85(3): 525-535.

2. Akhtar, A., M. Bishnoi, et al. (2020). "Sodium orthovanadate improves learning and memory in intracerebroventricular-streptozotocin rat model of Alzheimer's disease through modulation of brain insulin resistance induced tau pathology." Brain Research Bulletin 164: 83-97.

3. Ayoub, M. A. and K. D. Pfleger (2010). "Recent advances in bioluminescence resonance energy transfer technologies to study GPCR heteromerization." Current opinion in pharmacology. 10(1): 44-52.

4. Babiec, W. E., R. Guglietta, et al. (2014). "Ionotropic NMDA receptor signaling is required for the induction of long-term depression in the mouse hippocampal CA1 region." Journal of Neuroscience 34(15): 5285-5290.

5. Bai, L., S. Zhang, et al. (2019). "Brain-derived neurotrophic factor induces thioredoxin-1 expression through TrkB/Akt/CREB pathway in SH-SY5Y cells." Biochimie 160: 55-60.

6. Bai, M., X. Zhu, et al. (2012). "Abnormal hippocampal BDNF and miR-16 expression is associated with depression-like behaviors induced by stress during early life." PLoS One 7(10): e46921.

7. Bartos, J. and M. Pesez (1979). "Colorimetric and fluorimetric determination of aldehydes and ketones." Pure Appl Chem 51(8): 1803-1814.

8. Belleau, E. L., M. T. Treadway, et al. (2019). "The Impact of Stress and Major Depressive Disorder on Hippocampal and Medial Prefrontal Cortex Morphology." Biological Psychiatry 85(6): 443-453.

9. Bibring, E. (1953). "The mechanism of depression."

10. Bousman, C. A., K. Arandjelovic, et al. (2019). "Pharmacogenetic tests and depressive symptom remission: a meta-analysis of randomized controlled trials." Pharmacogenomics 20(01): 37-47.

11. Carroll, B. J. (1971). "Monoamine precursors in depression: Clinical trials and theoretical implications." Comments on Contemporary Psychiatry.

12. Chen, B., D. Dowlatshahi, et al. (2001). "Increased hippocampal bdnf immunoreactivity in subjects treated with antidepressant medication." Biological Psychiatry. 50(4): 260-265.

13. Cherry, J., A. Calder, et al. (2017). Small molecule approaches to upregulate SMN expression from the SMN2 locus. Spinal Muscular Atrophy, Elsevier: 283-299.

14. Cortese, G. P., R. M. Barrientos, et al. (2011). "Aging and a peripheral immune challenge interact to reduce mature brain-derived neurotrophic factor and activation of TrkB, PLCY1, and ERK in hippocampal synaptoneurosomes." Journal of Neuroscience 31(11): 4274-4279. 
15. de Sousa, R. T., M. V Zanetti, et al. (2015). "Challenging treatment-resistant major depressive disorder: a roadmap for improved therapeutics." Current neuropharmacology. 13(5): 616-635.

16. Duman, R. S., J. Malberg, et al. (2000). "Neuronal plasticity and survival in mood disorders." Biological psychiatry_ 48(8): 732-739.

17. Fawcett, J. and R. L. Barkin (1997). "Efficacy issues with antidepressants." The Journal of clinical psychiatry 58(suppl 6): 32-39.

18. Feng, H., C. Wang, et al. (2019). "Roflumilast ameliorates cognitive impairment in APP/PS1 mice via CAMP/CREB/BDNF signaling and anti-neuroinflammatory effects." Metabolic brain disease 34(2): 583-591.

19. Ferré, S., R. Baler, et al. (2009). "Building a new conceptual framework for receptor heteromers." Nature chemical biology_ 5(3): 131-134.

20. Frühauf-Perez, P. K., F. R. Temp, et al. (2018). "Spermine protects from LPS-induced memory deficit via BDNF and TrkB activation." Neurobiology of learning and memory 149: 135-143.

21. Fukuchi, M. (2020). "Identifying inducers of BDNF gene expression from pharmacologically validated compounds; antipyretic drug dipyrone increases BDNF mRNA in neurons." Biochemical and biophysical research communications 524(4): 957-962.

22. Gomes, C., R. Ferreira, et al. (2013). "Activation of microglial cells triggers a release of brain-derived neurotrophic factor (BDNF) inducing their proliferation in an adenosine A 2A receptor-dependent manner: $A$ $2 \mathrm{~A}$ receptor blockade prevents BDNF release and proliferation of microglia." Journal of neuroinflammation 10(1): 1-13.

23. Gomez, K., A. Calderón-Rivera, et al. (2020). "Cdk5-dependent phosphorylation of CaV3. 2 T-type channels: possible role in nerve ligation-induced neuropathic allodynia and the compound action potential in primary afferent C fibers." Journal of Neuroscience 40(2): 283-296.

24. Gornall, A. G., C. J. Bardawill, et al. (1949). "Determination of serum proteins by means of the biuret reaction." Journal of biological chemistry. 177(2): 751-766.

25. Harlow, L. L., M. D. Newcomb, et al. (1986). "Depression, self-derogation, substance use, and suicide ideation: Lack of purpose in life as a mediational factor." Journal of clinical psychology 42(1): 5-21.

26. Hasbi, A., T. Fan, et al. (2009). "Calcium signaling cascade links dopamine D1-D2 receptor heteromer to striatal BDNF production and neuronal growth." Proceedings of the National Academy of Sciences 106(50): 21377-21382.

27. Hasegawa, Y., H. Suzuki, et al. (2011). "Preservation of tropomyosin-related kinase B (TrkB) signaling by sodium orthovanadate attenuates early brain injury after subarachnoid hemorrhage in rats." Stroke 42(2): 477-483.

28. Hern, J. A., A. H. Baig, et al. (2010). "Formation and dissociation of M1 muscarinic receptor dimers seen by total internal reflection fluorescence imaging of single molecules." Proceedings of the National Academy of Sciences 107(6): 2693-2698.

29. Hutchinson, A. J., C.-L. Chou, et al. (2009). "Activation of EP2 prostanoid receptors in human glial cell lines stimulates the secretion of BDNF." Neurochemistry International 54(7): 439-446.

30. Idayu, N. F., M. T. Hidayat, et al. (2011). "Antidepressant-like effect of mitragynine isolated from Mitragyna speciosa Korth in mice model of depression." Phytomedicine 18(5): 402-407. 
31. Jacobsen, J. P. and A. Mørk (2006). "Chronic corticosterone decreases brain-derived neurotrophic factor (BDNF) mRNA and protein in the hippocampus, but not in the frontal cortex, of the rat." Brain Research 1110(1): 221-225.

32. Jollow, D., J. Mitchell, et al. (1974). "Bromobenzene-induced liver necrosis. Protective role of glutathione and evidence for 3, 4-bromobenzene oxide as the hepatotoxic metabolite." Pharmacology. 11(3): 151-169.

33. Karege, F., G. Vaudan, et al. (2005). "Neurotrophin levels in postmortem brains of suicide victims and the effects of antemortem diagnosis and psychotropic drugs." Molecular Brain Research 136(1-2): 29-37.

34. Katayama, K., K. Yamada, et al. (2010). "Slitrk1-deficient mice display elevated anxiety-like behavior and noradrenergic abnormalities." Molecular Psychiatry. 15(2): 177-184.

35. Kawano, T., K. Fukunaga, et al. (2001). "Neuroprotective effect of sodium orthovanadate on delayed neuronal death after transient forebrain ischemia in gerbil hippocampus." Journal of Cerebral Blood Flow \& Metabolism 21(11): 1268-1280.

36. Kim, U. J., B. H. Lee, et al. (2019). "Neuroprotective effects of a protein tyrosine phosphatase inhibitor against hippocampal excitotoxic injury." Brain Research 1719: 133-139.

37. Kono, Y. (1978). "Generation of superoxide radical during autoxidation of hydroxylamine and an assay for superoxide dismutase." Archives of biochemistry and biophysics 186(1): 189-195.

38. Kulkarni, S. and A. Dhir (2007). "Effect of various classes of antidepressants in behavioral paradigms of despair." Progress in Neuro-Psychopharmacology and Biological Psychiatry. 31(6): 1248-1254.

39. LeMoult, J. and I. H. Gotlib (2019). "Depression: A cognitive perspective." Clinical Psychology Review 69: 51-66.

40. Levi-Montalcini, R. (1950). The origin and development of the visceral system in the spinal cord of the chick embryo, World Scientific.

41. Liu, Q. R., D. Walther, et al. (2005). "Human brain derived neurotrophic factor (BDNF) genes, splicing patterns, and assessments of associations with substance abuse and Parkinson's Disease." American Journal of Medical Genetics Part B: Neuropsychiatric Genetics 134(1): 93-103.

42. Machado, D. G., M. P. Cunha, et al. (2012). "Fluoxetine reverses depressive-like behaviors and increases hippocampal acetylcholinesterase activity induced by olfactory bulbectomy." Pharmacology Biochemistry. and Behavior 103(2): 220-229.

43. Marshall, J., X.-z. Zhou, et al. (2018). "Antidepression action of BDNF requires and is mimicked by Gai1/3 expression in the hippocampus." Proceedings of the National Academy of Sciences 115(15): E3549-E3558.

44. McGill, B. E., S. F. Bundle, et al. (2006). "Enhanced anxiety and stress-induced corticosterone release are associated with increased <em>Crh</em> expression in a mouse model of Rett syndrome." Proceedings of the National Academy of Sciences 103(48): 18267-18272.

45. Mehrpouya, S., A. Nahavandi, et al. (2015). "Iron administration prevents BDNF decrease and depressivelike behavior following chronic stress." Brain Research 1596: 79-87.

46. Minichiello, L., A. M. Calella, et al. (2002). "Mechanism of TrkB-Mediated Hippocampal Long-Term Potentiation." Neuron 36(1): 121-137.

47. Mulinari, S. (2012). "Monoamine theories of depression: historical impact on biomedical research." Journal of the History of the Neurosciences 21(4): 366-392. 
48. Nagahara, A. H. and M. H. Tuszynski (2011). "Potential therapeutic uses of BDNF in neurological and psychiatric disorders." Nature reviews Drug discovery. 10(3): 209-219.

49. Nollet, M., A. M. L. Guisquet, et al. (2013). "Models of depression: unpredictable chronic mild stress in mice." Current protocols in pharmacology 61(1): 5.65. 61-65.65. 17.

50. Numakawa, T., H. Odaka, et al. (2017). "Actions of Brain-Derived Neurotrophic Factor and Glucocorticoid Stress in Neurogenesis." International journal of molecular sciences 18(11): 2312.

51. Schildkraut, J. J. (1965). "The catecholamine hypothesis of affective disorders: a review of supporting evidence." American journal of Psychiatry. 122(5): 509-522.

52. Schiller, C. E., J. Minkel, et al. (2013). "Remitted major depression is characterized by reduced prefrontal cortex reactivity to reward loss." Journal of Affective Disorders 151(2): 756-762.

53. Seifer, D. B., B. Feng, et al. (2006). "Immunocytochemical evidence for the presence and location of the neurotrophin-Trk receptor family in adult human preovulatory ovarian follicles." American journal of obstetrics and gynecology. 194(4): 1129-1134.

54. Sekar, N., A. Kanthasamy, et al. (1990). "Antioxidant effect of vanadate on experimental diabetic rats." Acta diabetologia latina 27(4): 285-293.

55. Sheikh, H. I., E. P. Hayden, et al. (2010). "Genotyping the BDNF rs6265 (val66met) polymorphism by onestep amplified refractory mutation system PCR." Psychiatric genetics 20(3): 109.

56. Trifilieff, P., M.-L. Rives, et al. (2011). "Detection of antigen interactions ex vivo by proximity ligation assay: endogenous dopamine D2-adenosine A2A receptor complexes in the striatum." Biotechniques 51(2): 111118.

57. Wang, W., Y. Lu, et al. (2015). "Rapid-acting antidepressant-like effects of acetyl-I-carnitine mediated by $\mathrm{PI3K} / \mathrm{AKT} / \mathrm{BDNF} / \mathrm{VGF}$ signaling pathway in mice." Neuroscience 285: 281-291.

58. Wang, Y., X.-L. Cui, et al. (2011). "LPS inhibits the effects of fluoxetine on depression-like behavior and hippocampal neurogenesis in rats." Progress in Neuro-Psychopharmacology and Biological Psychiatry. 35(8): 1831-1835.

59. Wills, E. (1965). "Mechanisms of lipid peroxide formation in tissues role of metals and haematin proteins in the catalysis of the oxidation of unsaturated fatty acids." Biochimica et Biophysica Acta (BBA)-Lipids and Lipid Metabolism 98(2): 238-251.

60. Wolkowitz, O. M., J. Wolf, et al. (2011). "Serum BDNF levels before treatment predict SSRI response in depression." Progress in Neuro-Psychopharmacology and Biological Psychiatry 35(7): 1623-1630.

61. Yang, L., Y. Zhao, et al. (2015). "The Effects of Psychological Stress on Depression." Current neuropharmacology_ 13(4): 494-504.

62. Zhang, X. Y., D.-C. Chen, et al. (2015). "The interplay between BDNF and oxidative stress in chronic schizophrenia." Psychoneuroendocrinology_ 51: 201-208.

\section{Figures}




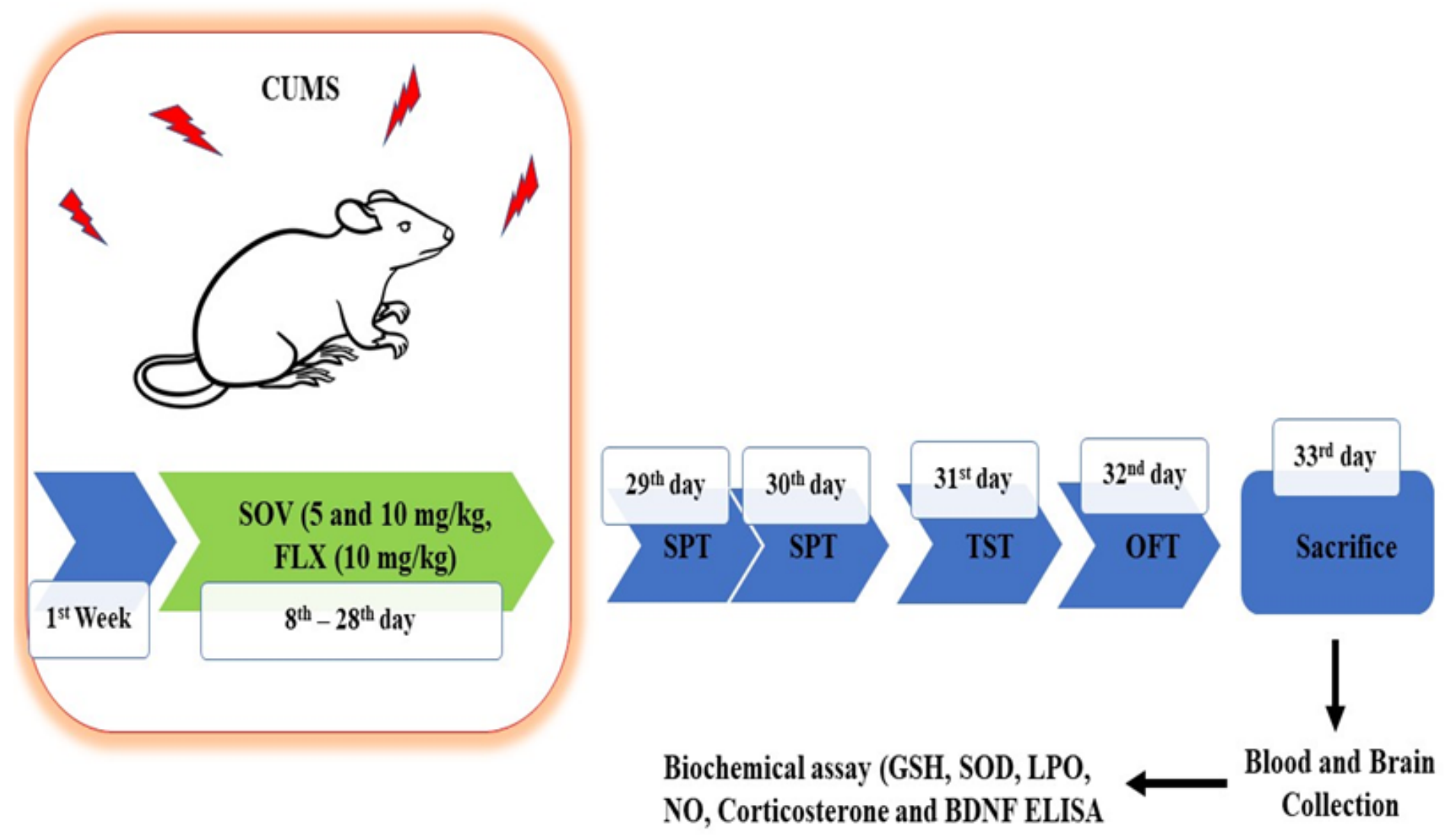

SPT: Sucrose preference test

TST: Tail suspension test

OFT: Open field test

*Body weight was analysed on weekly basis

\section{Figure 1}

Experimental design.

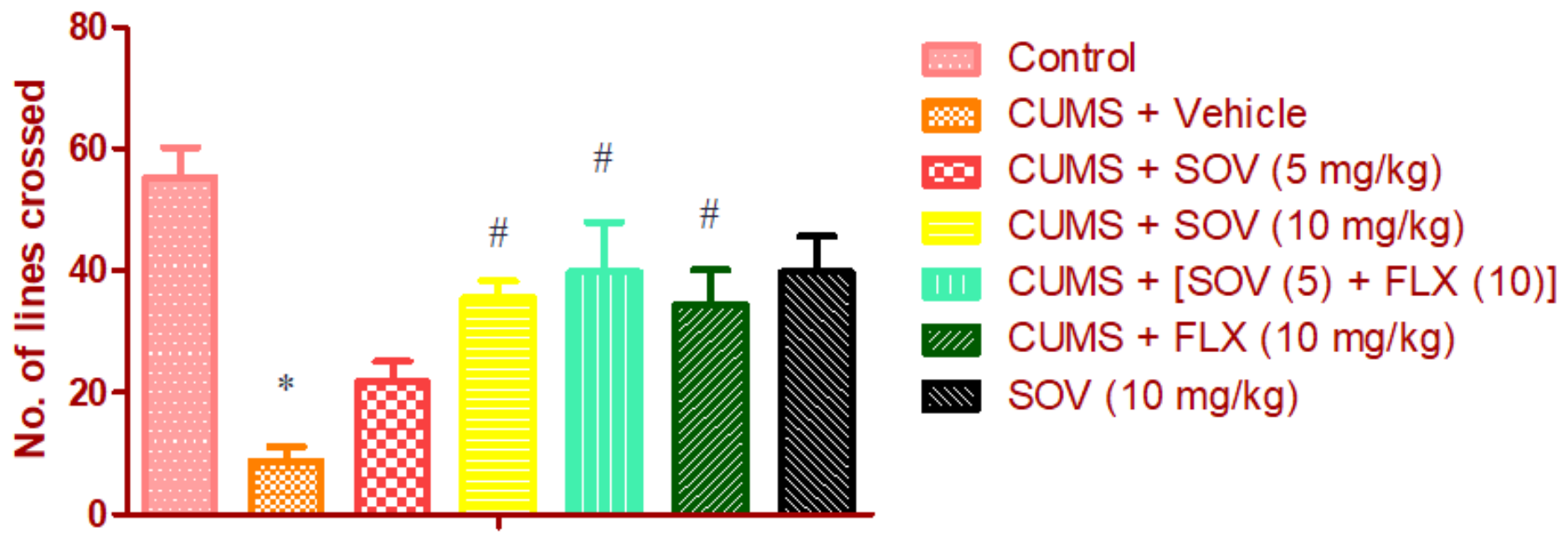


Figure 2

Effect of SOV, fluoxetine, and their combination on locomotor activity in the open field test. Values are expressed as mean \pm SEM. For statistical significance, ${ }^{*} p<0.05$ compared to the control group; $\# p<0.05$ compared to CUMS group (One-way ANOVA followed by Tukey's post hoc test). CUMS: Chronic Unpredictable Mild Stress, SOV: Sodium orthovanadate.

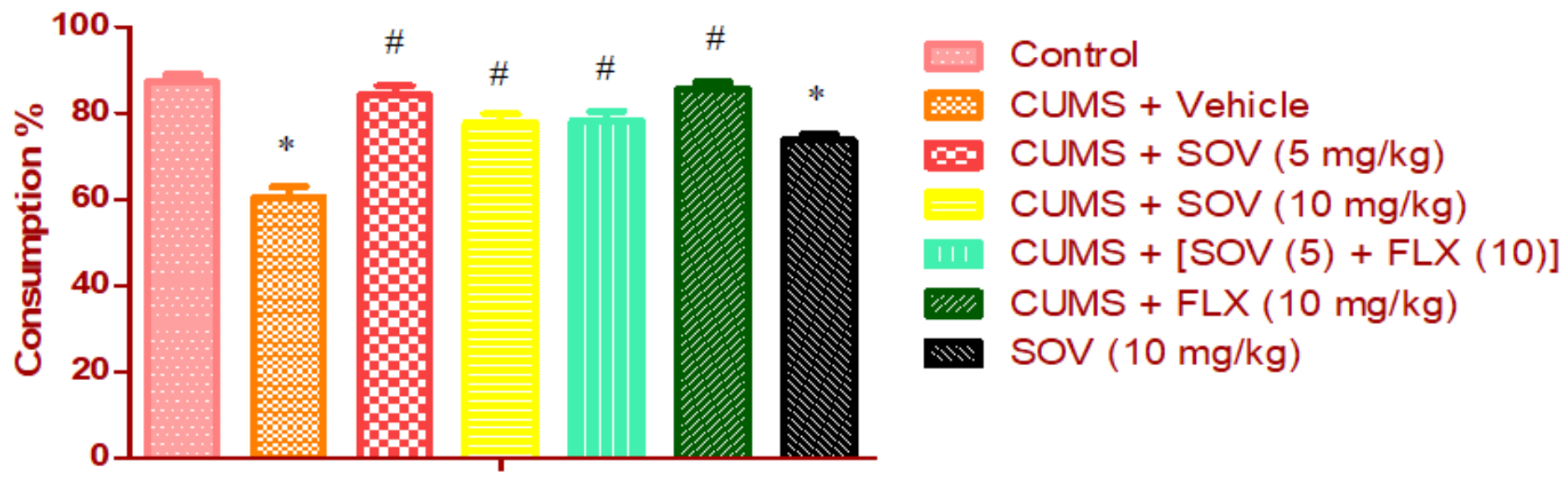

Figure 3

Effect of SOV, fluoxetine and their combination on the consumption of sucrose in sucrose preference test Values are expressed as mean \pm SEM. For statistical significance, ${ }^{*} p<0.05$ compared to the control group; $\# p<0.05$ compared to CUMS group (One-way ANOVA followed by Tukey's post hoc test). CUMS: Chronic Unpredictable Mild Stress, SOV: Sodium orthovanadate.

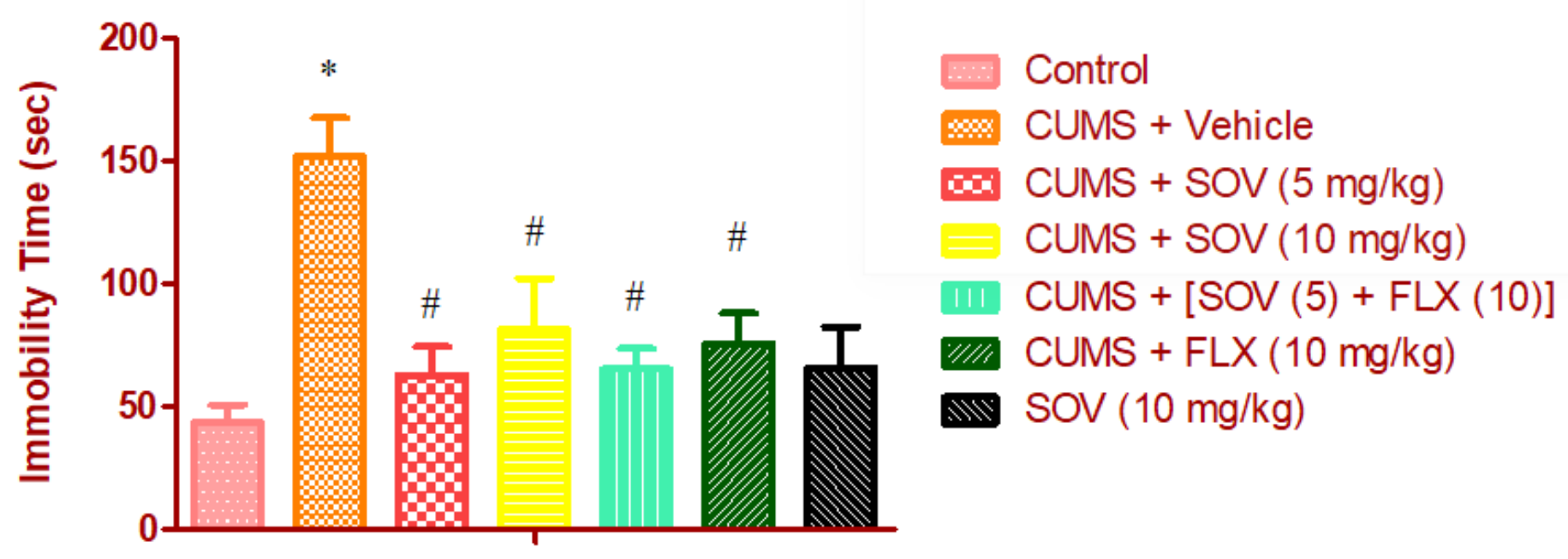

Figure 4

Effect of SOV, fluoxetine and their combination on an immobility duration in tail suspension test Values are expressed as mean \pm SEM. Statistical significance was ${ }^{*} p<0.05$ compared to the control group; $\# p<0.05$ 
compared to CUMS group (One-way ANOVA followed by Tukey's post hoc test). CUMS: Chronic Unpredictable Mild Stress, SOV: Sodium orthovanadate.
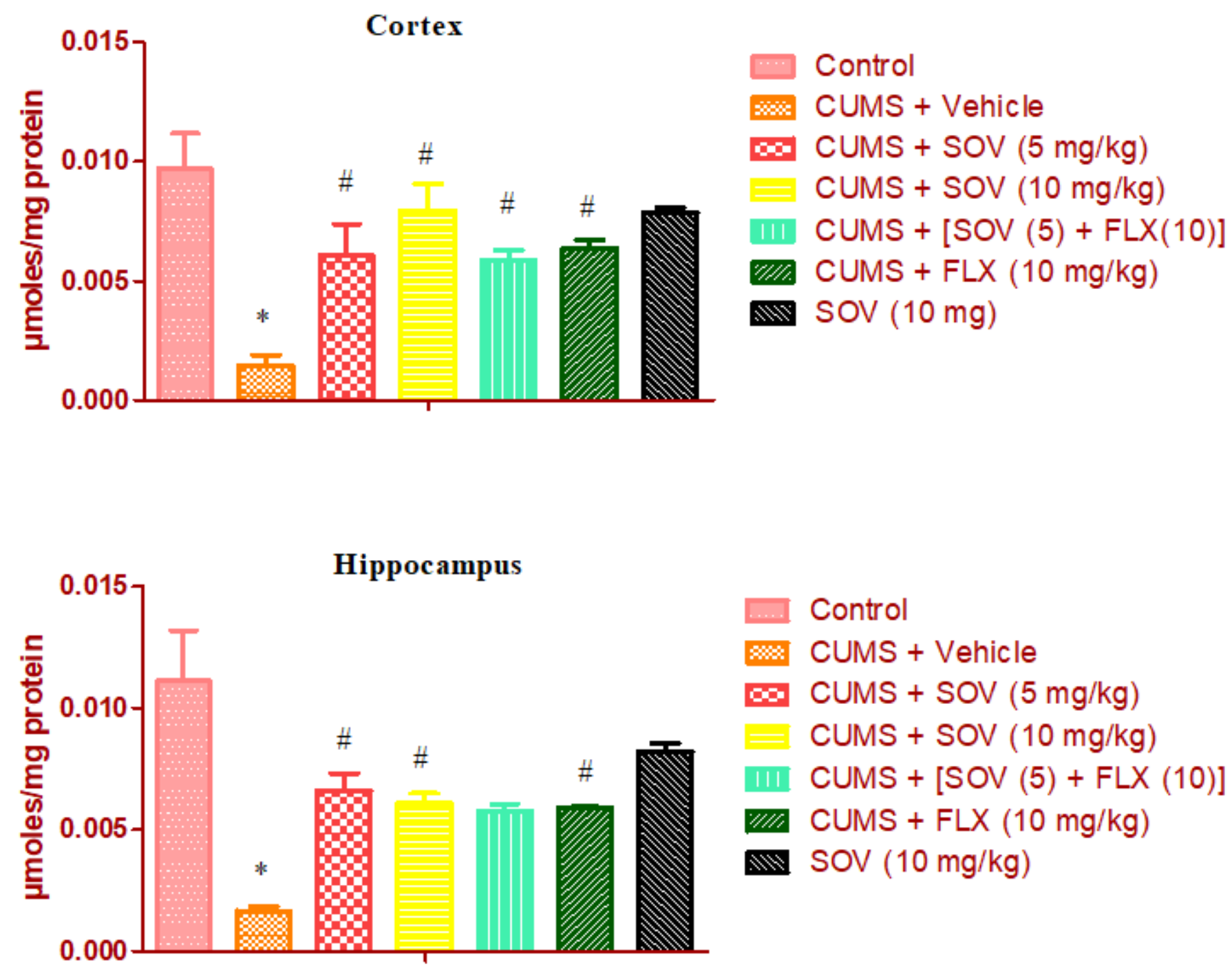

Figure 5

Effect of SOV, fluoxetine, and their combination on GSH levels in cortex and hippocampus of CUMS induced depressive mice. Values are expressed as mean $\pm \mathrm{SEM}$. For statistical significance, ${ }^{*} p<0.05$ compared to the control group; \#p<0.05 compared to CUMS group (One-way ANOVA followed by Tukey's post hoc test). CUMS: Chronic Unpredictable Mild Stress, SOV: Sodium Orthovanadate. 


\section{Cortex}

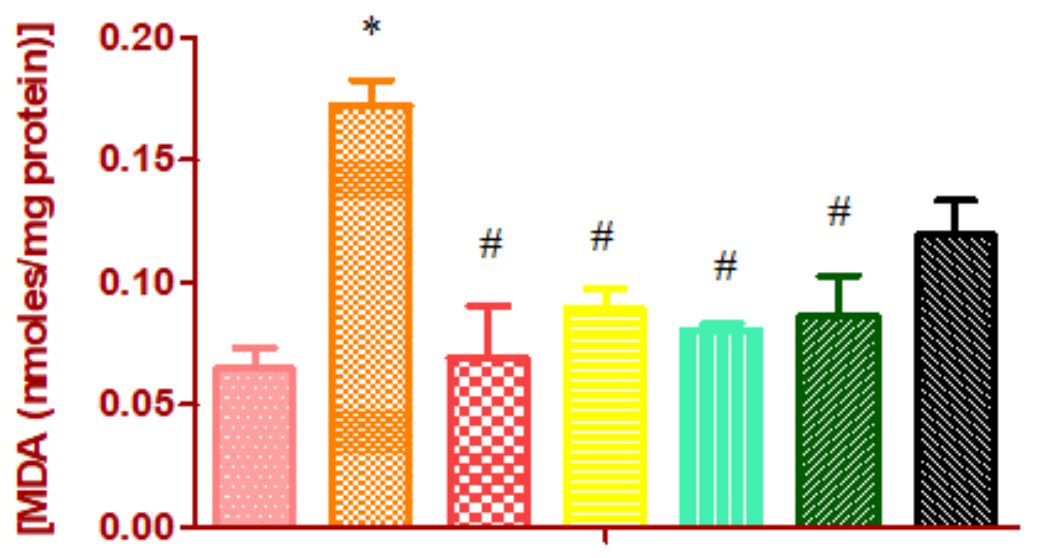

2.... Control

\# CUMS + Vehicle

0 CUMS + SOV $(5 \mathrm{mg} / \mathrm{kg})$

CUMS + SOV $(10 \mathrm{mg} / \mathrm{kg})$

III CUMS + [SOV (5) + FLX (10)]

WI. CUMS + FLX $(10 \mathrm{mg} / \mathrm{kg})$

aN SOV $(10 \mathrm{mg} / \mathrm{kg})$

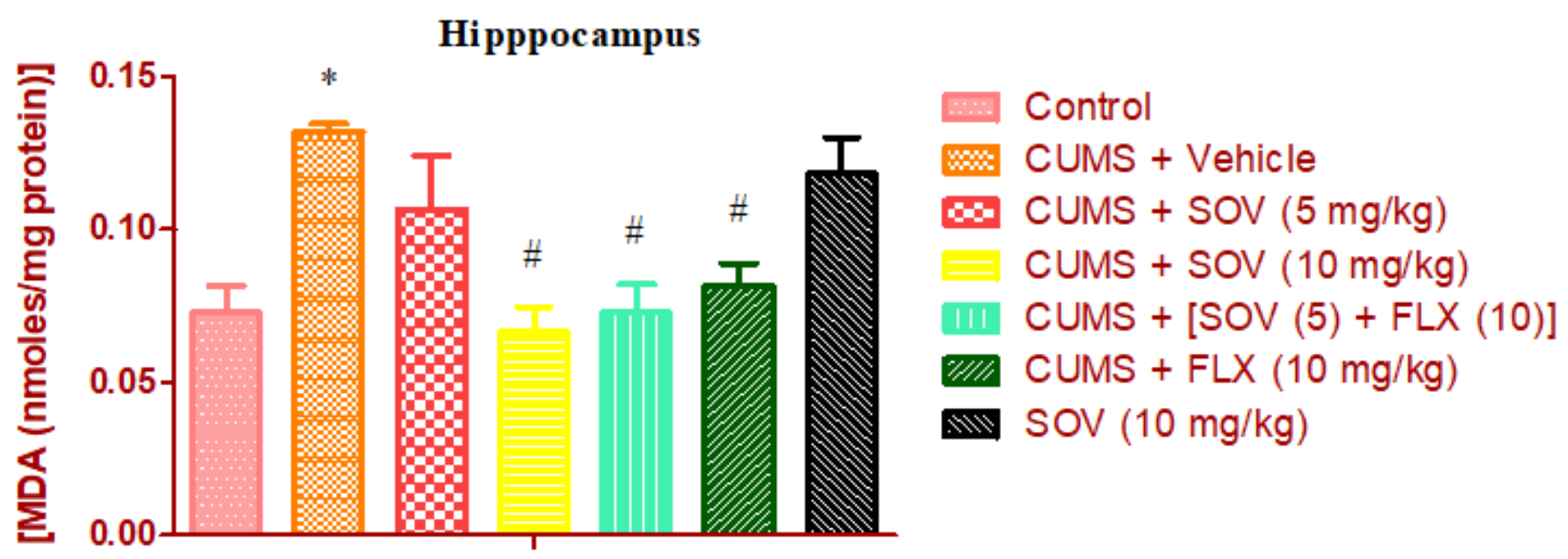

Figure 6

Effect of SOV, fluoxetine, and their combination on LPO levels in cortex and hippocampus of CUMS induced depressive mice. Values are expressed as mean \pm SEM. For statistical significance, ${ }^{*} p<0.05$ compared to the control group; $\# \mathrm{p}<0.05$ compared to CUMS group (One-way ANOVA followed by Tukey's post hoc test). CUMS: Chronic Unpredictable Mild Stress, SOV: Sodium Orthovanadate. 


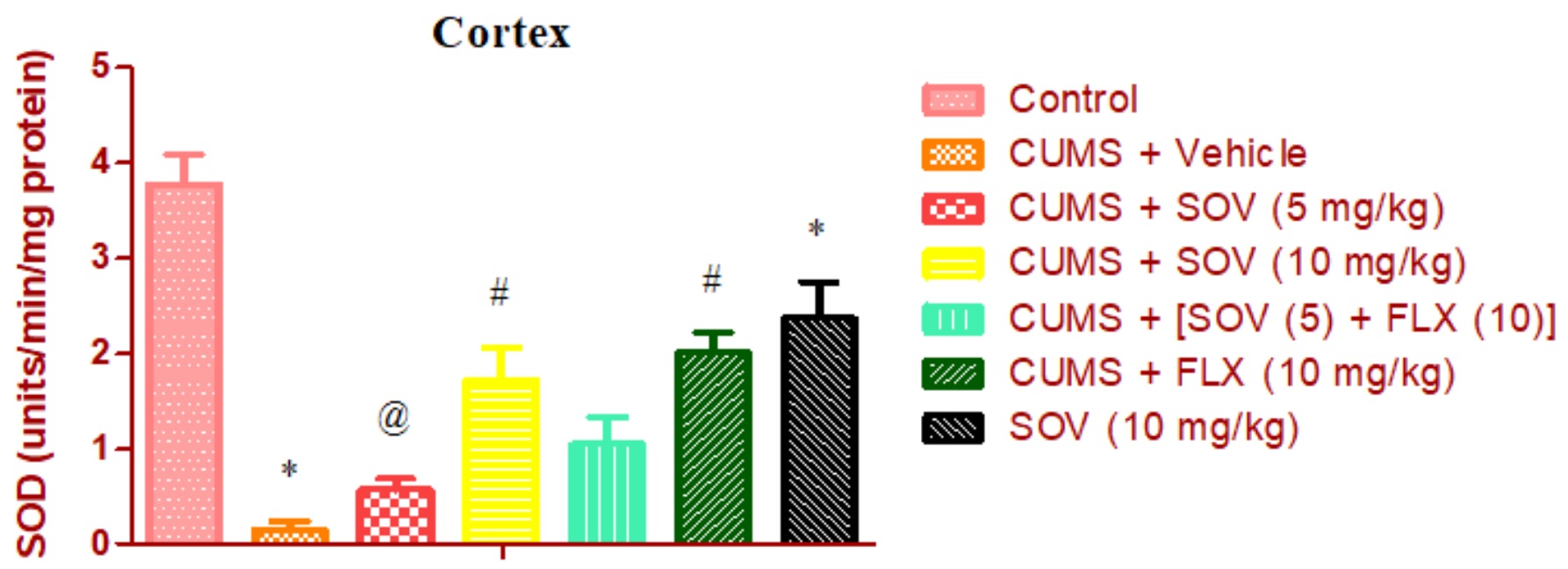

Hippocampus

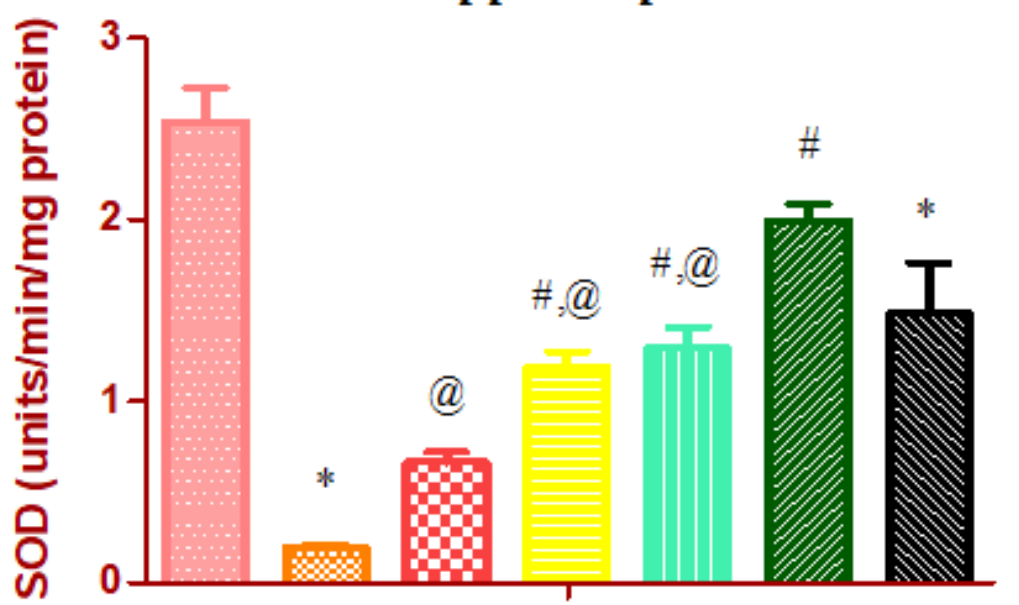
Control
CUMS + Vehicle
B CUMS + SOV $(5 \mathrm{mg} / \mathrm{kg})$
CUMS + SOV $(10 \mathrm{mg} / \mathrm{kg})$
III CUMS + [SOV (5) + FLX (10)]
III/. CUMS + FLX (10 mg/kg)
AIV SOV $(10 \mathrm{mg} / \mathrm{kg})$

\section{Figure 7}

Effect of SOV, fluoxetine, and their combination on the activity of SOD in cortex and hippocampus of CUMS induced depressive mice. Values are expressed as mean \pm SEM. For statistical significance, ${ }^{*} p<0.05$ as compared to the control group; \#p $<0.05$ as compared to CUMS group, @p $<0.05$ as compared to fluoxetine (Oneway ANOVA followed by Tukey's post hoc test). CUMS: Chronic Unpredictable Mild Stress, SOV: Sodium Orthovanadate. 


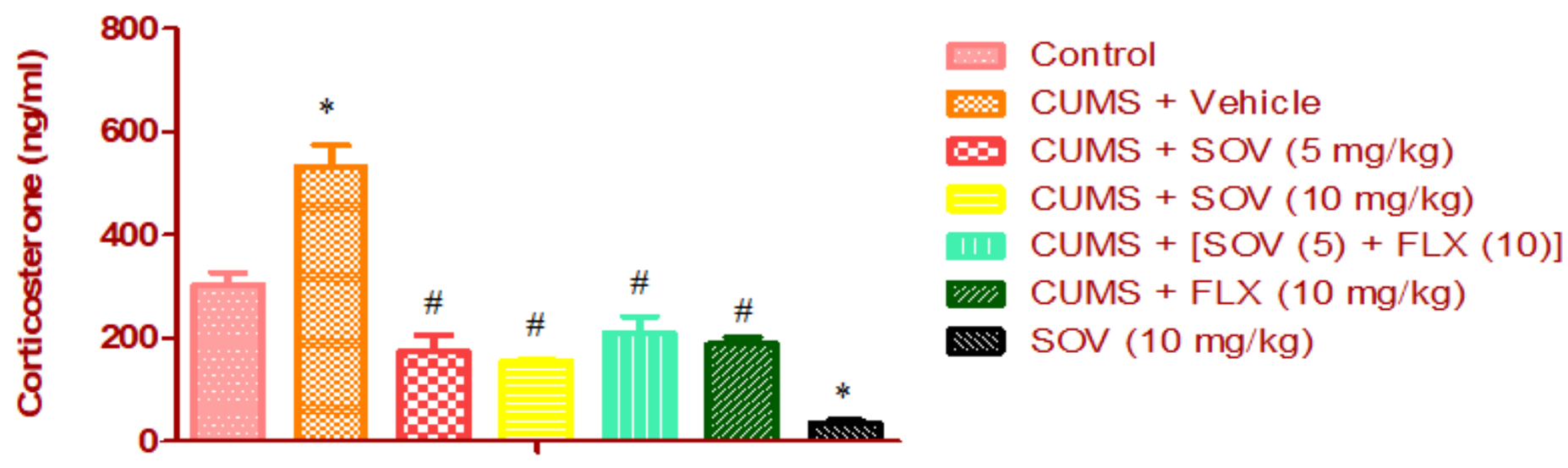

Figure 8

Effect of SOV, fluoxetine, and their combination on corticosterone levels in the plasma of CUMS induced depressive mice. Values are expressed as mean \pm SEM. For statistical significance, ${ }^{*} p<0.05$ compared to the control group; \#p<0.05 compared to CUMS group (One-way ANOVA followed by Tukey's post hoc test). CUMS: Chronic Unpredictable Mild Stress, SOV: Sodium Orthovanadate. 

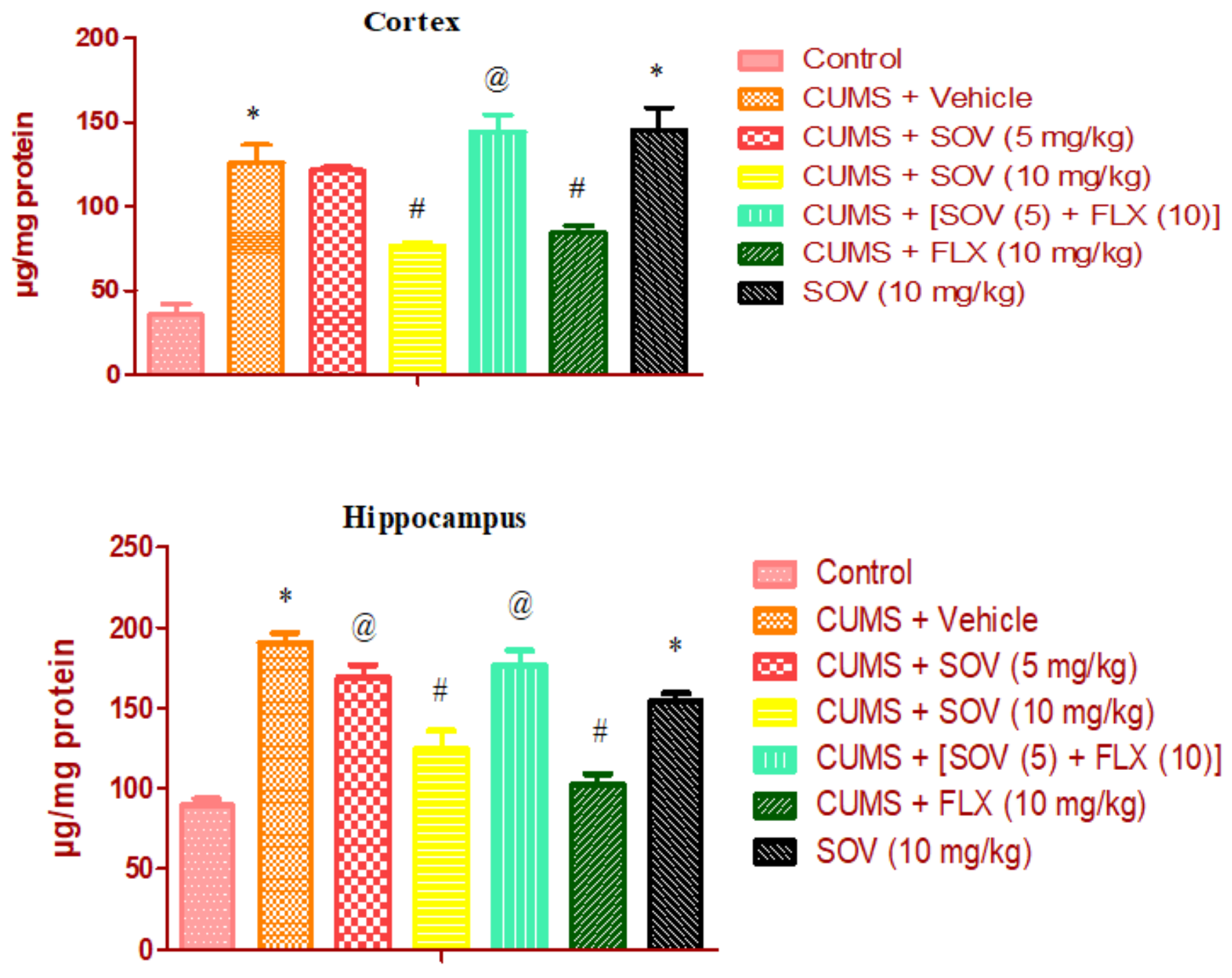

Figure 9

Effect of SOV, fluoxetine, and their combination on the levels of NO in cortex and hippocampus of CUMS induced depressive mice. Values are expressed as mean \pm SEM. For statistical significance, ${ }^{\star} p<0.05$ as compared to the control group; \#p<0.05 as compared to CUMS group, @p $<0.05$ as compared to fluoxetine (Oneway ANOVA followed by Tukey's post hoc test). CUMS: Chronic Unpredictable Mild Stress, SOV: Sodium Orthovanadate. 

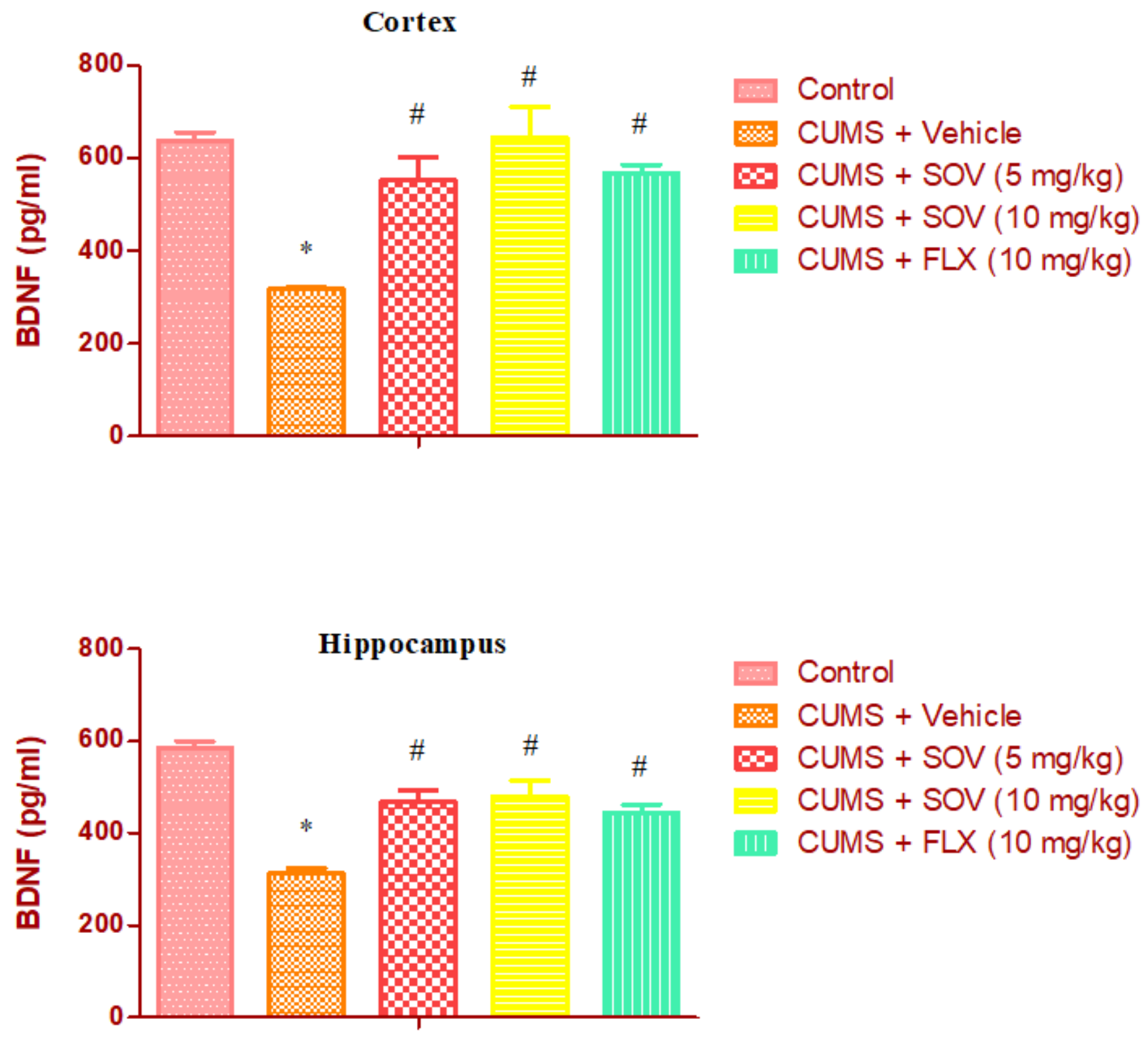

Figure 10

Effect of SOV and fluoxetine on the levels of BDNF in cortex and hippocampus. Values are expressed as mean \pm SEM. For statistical significance, ${ }^{*} p<0.05$ compared to the control group; $\# p<0.05$ compared to CUMS group (One-way ANOVA followed by Tukey's post hoc test). CUMS: Chronic Unpredictable Mild Stress, SOV: Sodium Orthovanadate. 